\title{
Clinical Analysis of 186 Cases of Postpartum Hemorrhage after Cesarean Section
}

\author{
Xianyan $\mathbf{F u}^{*}$ \\ Maternal and Child Health Care Hospital of Qidong County, Hengyang, Hunan 421600, China
}

\begin{abstract}
Objective: In this paper, we analyzed retrospectively 186 cases of postpartum hemorrhage in our hospital, and analyzed the characteristics and clinical analysis of postpartum hemorrhage in cesarean section. Method: Pregnant women were selected from March 2010 to March 2014 in our hospital. The causes of postpartum hemorrhage and postpartum hemorrhage were compared with natural labor and cesarean section. Results: In 4622 pregnant women, 2631 cases are cesarean section while 1991 cases are vaginal delivery. There were 268 cases of postpartum hemorrhage, including 82 cases of postpartum vaginal bleeding, 186 cases of postpartum hemorrhage after cesarean section. The incidence of postpartum hemorrhage was $7.07 \%$, and the rate vaginal birth postpartum hemorrhage was $4.11 \%$, and there was significant difference between them $(p<0.01)$. Uterine inertia is two kinds of mode of delivery to the main causes of postpartum hemorrhage, followed by rupture of the soft birth canal and placental factors etc. Conclusion: Cesarean section is the cause of postpartum hemorrhage. It was found that pregnant women postpartum hemorrhage should actively take treatment to ensure the safety of pregnant women in perinatal period.
\end{abstract}

\section{KEYWORDS}

Cesarean section

Postpartum hemorrhage

Clinical analysis

\section{Introduction}

Postpartum hemorrhage is one of the common complications of cesarean section, and it is an important aspect to guarantee the safety of maternal production. Cesarean section is an effective solution to solve the problem, but postpartum hemorrhage often affects the prognosis of pregnant women and postpartum recovery, and even critical pregnant women's life safety. In this paper, we analyzed retrospectively 186 cases of postpartum hemorrhage in our hospital, and analyzed the characteristics and clinical analysis of postpartum hemorrhage in cesarean section.

\section{Materials and methods \\ 2.1. General information}

Copyright $\odot 2014$ Xianyan Fu

doi: $10.18686 /$ aem.v3i1.1

Received: February 19, 2014; Accepted: April 8, 2014; Published online: June 20, 2014

This is an open-access article distributed under the terms of the Creative Commons Attribution Unported License (http://creativecommons.org/ licenses/by-nc/4.0/), which permits unrestricted use, distribution, and reproduction in any medium, provided the original work is properly cited.

${ }^{\star}$ Corresponding author: Maternal and Child Health Care Hospital of Qidong County, Hengyang, Hunan 421600, China. E-mail: xianyan_906@163.com
A total of 4622 pregnant women from March 2010 to March 2014 were selected, including 2631 cases of cesarean section, 1991 cases of vaginal delivery where 268 cases has postpartum hemorrhage. Pregnant women who aged between 23 to 37 years old were selected where the weeks of pregnant is around 35 to 42 weeks, the average gestational age is 37.2 weeks. There were 138 cases of pregnant women in the first time, two or more of the 48 cases of maternal. Following the approval of the ethics committee of the hospital and the patient informed consent, the clinical data of all patients were analyzed by clinical data.

\subsection{Diagnostic criteria for postpartum hemorrhage}

The diagnostic criteria of postpartum hemorrhage: In the 2 hours after birth, the amount of bleeding is greater than $400 \mathrm{~mL}$; or the amount of bleeding within 24 hours after the production of the mother can be considered as postpartum hemorrhage [1].

\subsection{Cesarean section postpartum blood volume measurement method}

For cesarean section, the negative pressure collecting dish is mainly used to suck the amniotic fluid collected after operation. The amount of the bleeding and gauze volume using intrapartum gauze weighing was calculated and 
compared the results of with the three of the total amount of blood loss. The statistics of the amount of bleeding in the vaginal delivery of pregnant women mainly uses the capacity method, with the weight method and the area method. After the delivery of the baby, the poly blood basin is placed at the women's hips to collect the amount of bleeding, the method of same area calculation of gauze and single blood production, perineal pad of increased blood volume, pregnant women with a total amount of bleeding.

\subsection{Statistical processing}

All the data in this study were processed by SPSS 17.0 statistical software, and the measurement data using mean \pm standard deviation $(\bar{x} \pm s)$. The count data rates were expressed as percentage (\%), when $p<0.05$ indicates a statistically significant difference.

\section{Results}

In 4622 pregnant women there were 2631 cases of cesarean section and 1991 cases of vaginal delivery. From that, there were 268 cases of postpartum hemorrhage, including 82 cases of vaginal bleeding, 186 cases of postpartum hemorrhage after cesarean section. The incidence of postpartum hemorrhage was $7.07 \%$, and the rate of postpartum vaginal bleeding was $4.11 \%$, and there was significant difference between them $(p<0.01)$.

\subsection{Causes of postpartum hemorrhage}

Comparing the causes of hemorrhage after vaginal delivery and cesarean section, the specific reasons and statistical data are as follows.

According to the table, we can see, two kinds of mode of delivery leads to the main cause of postpartum hemorrhage is uterine inertia, followed by soft birth canal rupture and placental factors.

\subsection{Analysis of the causes of postpartum hemor- rhage in cesarean section \\ 3.2.1. Uterine inertia}

There are 91 cases of pregnant women due to uterine contraction caused by fatigue and postpartum hemorrhage. The uterine excessive expansion in 38 cases has accounting for $41.8 \%$, resulting in uterine excessive expansion of reasons baby, twins or multiple births, polyhydramnios. In addition, there were 29 cases for uterine scar, accounted for $31.9 \%$; pregnancy induced hypertension in 15 cases, accounted for $16.5 \%$; other 9 cases accounted for $9.9 \%$.

\subsubsection{Placenta factor}

A total of 38 cases of placental factors leading to postpartum hemorrhage. There are 20 cases in which the placenta previa, accounting for 51.3\%; placental adherence 9 cases, accounting for $23.7 \%$; 4 cases of placenta accreta, accounting for $10.5 \%$; placental abruption 1 cases, accounting for $2.63 \%$; residual placenta 4 cases, accounting for $10.5 \%$

\subsection{Cesarean section postpartum hemorrhage and cesarean section indication analysis}

Cesarean section surgical indications in 41 cases of placenta previa, bleeding was $1635 \pm 1326 \mathrm{~mL}, 30$ cases in prenatal extend/stagnation, the blood loss was $1328 \pm 1023 \mathrm{~mL}$, head basin asymmetric is 29 cases which blood loss was $1045 \pm 865 \mathrm{~mL}$ tire Road distress is 25 cases and the blood loss was $1023 \pm 569 \mathrm{~mL}$, uterine scar is 13 cases where

Table 1. Comparison of the causes of postpartum hemorrhage in the vaginal delivery and cesarean section.

\begin{tabular}{|c|c|c|c|c|c|}
\hline Group & $\mathrm{n}$ & Uterine inertia & The soft birth canal rupture & Placenta factor & Other \\
\hline Vaginal delivery group & 82 & 32 (39.0\%) & 29 (35.4\%) & 13 (15.9\%) & $8(9.76 \%)$ \\
\hline Cesarean section & 18 & $91(48.9 \%)$ & $46(24.7 \%)$ & $38(20.4 \%)$ & $11(5.91 \%)$ \\
\hline
\end{tabular}

Table 2. Analysis of the indications for cesarean section postpartum hemorrhage and cesarean section.

\begin{tabular}{cccc}
\hline Operative indication & $\mathrm{n}$ & Postpartum hemorrhage ratio (\%) & Average bleeding volume (ml) \\
\hline Placenta & 41 & 22.04 & $1635 \pm 1326$ \\
Extension / stagnation & 30 & 16.12 & $1328 \pm 1023$ \\
Head basin asymmetry & 29 & 15.60 & $1045 \pm 865$ \\
Fetal distress & 25 & 13.44 & $1023 \pm 569$ \\
Scar uterus & 13 & 6.99 & $895 \pm 398$ \\
Abnormal fetal position & 27 & 14.52 & $856 \pm 298$ \\
Multiple pregnancy & 10 & 5.38 & $798 \pm 296$ \\
Huge children & 5 & 2.69 & $716 \pm 201$ \\
Hypertensive disorders in pregnancy & 5 & 2.69 & $698 \pm 198$ \\
Induced abortion & 2 & 1.08 & $685 \pm 182$ \\
Gestational diabetes mellitus & 2 & 1.08 & $600 \pm 98$
\end{tabular}


blood loss was $895 \pm 398 \mathrm{~mL}$, fetal abnormalities in 27 case and blood loss was $856 \pm 298 \mathrm{~mL}$, multiple pregnancies 10 cases were blood loss was $798 \pm 296 \mathrm{~mL}$, huge children 5 cases, the amount of bleeding was $716 \pm 201 \mathrm{~mL}, 5$ cases in gestational hypertension, blood loss was $698 \pm 182 \mathrm{~mL}$, induction failure is 2 cases, blood loss was $385 \pm 182 \mathrm{~mL}, 2$ cases of gestational diabetes, blood loss was $600 \pm 98 \mathrm{~mL}$. Among the largest and the highest rate of hemorrhage was the placenta. Compared with others, the difference was statistically significant $(p<0.05)$. The amount of bleeding for labor to extend/stagnation, head basin asymmetry, fetal distress, scar uterus and fetal position abnormalities in these types of situations was analyzed as in Table 2 .

Because of the patients with low amniotic fluid in patients with early stripping operation, so the amount of bleeding not included in the statistics.

\section{Discussion}

Postpartum hemorrhage is the main cause of maternal death, but also one of the critical diseases of Obstetrics and Gynecology. With the improvement of the medical level, the cesarean section is the effective solution to the knowledge of the decision, and the safety of the section is gradually increasing. However, the incidence of postpartum hemorrhage in cesarean section is still higher than that of natural childbirth. The results of this study are also consistent with the clinical phenomenon, in 1991 cases of pregnant women, 82 cases of vaginal bleeding, the incidence rate of postpartum hemorrhage was $4.11 \%$; in 2631 cases of cesarean section, 186 cases of postpartum hemorrhage, the incidence rate was $7.07 \%$. It is one of the main reasons that cause the postpartum hemorrhage [2].

The cause of postpartum hemorrhage is more. According to the results of this study, the main cause of postpartum hemorrhage is uterine contraction stress. Because the pregnant women in the postnatal mainly rely on the uterine muscle contraction to stop bleeding, once the uterine muscle contraction capacity decreased it will inevitably make the pregnant woman bleeding phenomenon. There are many factors that affect the uterine contraction, such as pregnant women's mental state of prenatal stress and lead to rest is not sufficient or the process is too long and make physical decline. This is a muscle weakness phenomenon. In addition to the production process of anesthetic will also affect the uterine contraction ability of pregnant women. Therefore, for the above reasons, it should be in the production of pregnant women to prevent the preparation work, reduce the incidence of postpartum hemorrhage. For example, advising pregnant women to take full rest and do not have to worry too much about the tension, especially for the initial production of women. In addition to the production process of pregnant women is by controlling the dosage of narcotic drugs in order to avoid the phenomenon of uterine muscle fatigue caused by anesthesia drugs. Therefore clinical choice will position the cut in the lower segment of the uterus, and in the process of operation need to control the speed of fetal and fetal head. Taijian and carcass delivery time interval should be separated by about a minute or so, where let natural shedding of the placenta [3], and appropriate use of uterine contraction.

The other placenta factor is one of the causes of postpartum hemorrhage. According to the results of this study, the placenta is the main cause of postpartum hemorrhage. The causes of postpartum hemorrhage is due to the weakness of the muscular wall of the placenta, but the small blood vessels are abundant, and the external pressure of the bleeding caused by the bleeding is not enough. Generally, clinical treatment of postpartum hemorrhage is caused by the use of uterine cavity gauze packing method to stop bleeding. Another extraordinary flow history, the history of induced abortion of pregnant women easily leads to adherent placenta, placenta previa whereas placental spalling is not congruent in postpartum hemorrhage. The extraordinary history of patients who has the bleeding volume tends to be higher than those with no history of artificial abortion of pregnant women [4]. Therefore, the type of pregnant women should be prepared for the treatment of bleeding before surgery.

In prevention of postpartum hemorrhage, we need to strengthen the health education for pregnant women. A lot of pregnant women are often mistakenly think that the cesarean section is safe and less painful than natural childbirth, so the automatic requirement for cesarean section. In fact, caesarean section itself is the result in [5] the risk factors of postpartum hemorrhage, so when pregnant women choose to mode of production and need to correctly grasp cesarean section surgical indications for cesarean section. In addition, prenatal examination is very necessary. Prenatal examination can be found to be the cause of postpartum hemorrhage where can timely give prevention and treatment measures to reduce the occurrence of postpartum hemorrhage. Timely detection of maternal postpartum hemorrhage and actively take treatment are necessary to ensure the safety of pregnant women in perinatal period.

\section{References}

1. Le J. Obstetrics and Gynecology seventh edition. Beijing: People's medical publishing house. 2008:225-229.

2. East C. 10 years late postpartum hemorrhage clinical analysis. Practical Obstetrics and Gynecology. 2001;5(17):156.

3. Yang $\mathrm{H}, \mathrm{Ji}$ Z. Postpartum hemorrhage and clinical analysis. Chinese Medicine Oration. 2010:3.2(3):88.4.

4. Zhou Q. Gold W. Drug abortion on primipar's pregnancy, delivery and fetal effects. Modern Obstetrics And Gynecology Progress. 2000;9(1).

5. Magann EF, Evans S, Hutchinson M, et al. Postpartum hemorrhage after cesarean delivery: an analysis of risk factors. South Med J. 2005:98:681-685. 